

\title{
Boundary control synthesis for hyperbolic systems: A singular perturbation approach
}

Ying Tang, Christophe Prieur, Antoine Girard

\section{To cite this version:}

Ying Tang, Christophe Prieur, Antoine Girard. Boundary control synthesis for hyperbolic systems: A singular perturbation approach. CDC 2014 - 53rd IEEE Conference on Decision and Control, Dec 2014, Los Angeles, United States. pp.2840-2845, 10.1109/CDC.2014.7039825 . hal-01144135

\section{HAL Id: hal-01144135 \\ https://hal.science/hal-01144135}

Submitted on 21 Apr 2015

HAL is a multi-disciplinary open access archive for the deposit and dissemination of scientific research documents, whether they are published or not. The documents may come from teaching and research institutions in France or abroad, or from public or private research centers.
L'archive ouverte pluridisciplinaire HAL, est destinée au dépôt et à la diffusion de documents scientifiques de niveau recherche, publiés ou non, émanant des établissements d'enseignement et de recherche français ou étrangers, des laboratoires publics ou privés. 


\title{
Boundary control synthesis for hyperbolic systems: a singular perturbation approach
}

\author{
Ying TANG, Christophe PRIEUR and Antoine GIRARD
}

\begin{abstract}
In this paper, we consider the problem of boundary control of a class of linear hyperbolic systems of conservation laws based on the singular perturbation method. The full hyperbolic system is written as two subsystems, namely the reduced system representing the slow dynamics and the boundary-layer system standing for the fast dynamics. By choosing the boundary conditions for the reduced system as zero, the slow dynamics is stabilized in finite time. The main result is illustrated with a design of boundary control for a linearized Saint-Venant-Exner system. The stabilization of the full system is achieved with different boundary conditions for the fast dynamics.
\end{abstract}

\section{INTRODUCTION}

Many distributed physical systems are described by hyperbolic PDEs. This class of systems with infinite dimensional dynamics is relevant for a wide range of physical systems having an engineering interest, for instance, hydraulic networks for irrigation or navigation [1], gas flow in pipelines [2], networks of electrical transmission [3] or road traffic networks [4]. The significant importance of these applications motivates many research works on optimal control and controllability of hyperbolic systems as considered in [5], [6], [7].

The singular perturbation techniques started at the beginning of the 20th century. A great deal of the early motivation in this area arose from the studies of physical problems exhibiting both fast and slow dynamics, for instance DCmotor model, voltage regulator in [8]. In late 1980s, the research works in the singularly perturbed partial differential equations occurred. This kind of systems is interesting for analysis because of its relevance to many important phenomenon in different domains, as reported in the survey paper [9] where a comprehensive bibliography is involved.

The present paper focuses on the boundary control of linear hyperbolic systems. Our main contribution here is to achieve the boundary control synthesis using singular perturbation method. The full hyperbolic system of conservation laws is decomposed into two subsystems, the reduced system and the boundary-layer system. By selecting $K_{r}=0$ as the boundary conditions matrix for the reduced system, the slow dynamics converges to the origin in finite time. The boundary conditions matrix $K$ for the full system can be chosen such

Y. TANG and C. PRIEUR are with Department of Automatic Control, Gipsa-lab, 11 rue des Mathematiques, BP 46, 38402 Saint Martin d'Heres, France, ying.tang@gipsa-lab.fr, christophe.prieuragipsa-lab. fr.

A. GIRARD is with Department of Automatic Control, Gipsa-lab, 11 rue des Mathematiques, BP 46, 38402 Saint Martin d'Heres, France, and Laboratoire Jean Kuntzmann, Université de Grenoble, B.P. 53, 38041 Grenoble, France. antoine.girardeimag. fr. that the stability condition of the full dynamics is satisfied. Using Tikhonov theorem in [10], the full system converges to a small neighborhood of the equilibrium in finite time.

In this paper, the main result is applied to the SaintVenant-Exner model for the regulation of the water level in a channel. This problem has attracted the attention of many researchers for a long time, for instance in [11], [12] where the Lyapunov methods are used to stabilize such systems. In [13] the robust boundary control is designed for Saint-Venant equations with small perturbations.

The paper is outlined as follows. Section II recalls the class of singularly perturbed systems of conservation laws and the Tikhonov theorem for linear hyperbolic systems. In Section III, the boundary controller established in [10] is synthesized by the singular perturbation method. More precisely, when the boundary condition of the reduced system is chosen as zero, then the slow dynamics of the singularly perturbed system converges to the equilibrium in finite time. In Section IV, the main result is illustrated by an application, that is the design of boundary controls for the Saint-Venant-Exner equations. Finally, concluding remarks end the paper. Due to space limitation, some proofs have been omitted.

Notation. Given a matrix $G, G^{-1}$ and $G^{T}$ represent the inverse and the transpose matrix of $G$ respectively. For a symmetric matrix $S$, the minimum eigenvalue of the matrix $S$ is denoted by $\lambda_{\min }(S)$. For a positive integer $n, I_{n}$ is the identity matrix in $\mathbb{R}^{n \times n}$. || denotes the usual Euclidean norm in $\mathbb{R}^{n}$ and \|\| is associated with the matrix norm. \|\|$_{L^{2}}$ denotes the associated norm in $L^{2}(0,1)$ space, defined by $\|f\|_{L^{2}}=\left(\int_{0}^{1}|f|^{2} d x\right)^{\frac{1}{2}}$ for all functions $f \in L^{2}(0,1)$. Similarly, the associated norm in $H^{2}(0,1)$ space is denoted by \|\|$_{H^{2}}$, defined for all functions $h \in H^{2}(0,1)$, by $\|h\|_{H^{2}}=\left(\int_{0}^{1}|h|^{2}+\left|h_{x}\right|^{2}+\left|h_{x x}\right|^{2} d x\right)^{\frac{1}{2}}$. Following [14], we introduce the notation, for all matrices $K \in \mathbb{R}^{(n+m) \times(n+m),}$

$$
\rho_{1}(K)=\inf \left\{\left\|\Delta K \Delta^{-1}\right\|, \Delta \in D_{(n+m),+}\right\},
$$

where $D_{(n+m),+}$ denotes the set of diagonal positive matrix in $\mathbb{R}^{(n+m) \times(n+m)}$.

\section{LINEAR SINGULARLY PERTURBED SYSTEM OF CONSERVATION LAWS}

We consider the following singularly perturbed system of conservation laws for a small positive perturbation parameter $\varepsilon$

$$
\begin{aligned}
y_{t}(x, t)+\Lambda_{1} y_{x}(x, t) & =0, \\
\varepsilon z_{t}(x, t)+\Lambda_{2} z_{x}(x, t) & =0,
\end{aligned}
$$


where $x \in[0,1], t \in[0,+\infty), y:[0,1] \times[0,+\infty) \rightarrow \mathbb{R}^{n}$, $z:[0,1] \times[0,+\infty) \rightarrow \mathbb{R}^{m}, \Lambda_{1}$ and $\Lambda_{2}$ are diagonal positive matrices in $\mathbb{R}^{n \times n}$ and $\mathbb{R}^{m \times m}$ respectively.

The boundary conditions for system (1) are written as follows,

$$
\left(\begin{array}{l}
y(0, t) \\
z(0, t)
\end{array}\right)=K\left(\begin{array}{l}
y(1, t) \\
z(1, t)
\end{array}\right), \quad t \in[0,+\infty)
$$

where $K=\left(\begin{array}{ll}K_{11} & K_{12} \\ K_{21} & K_{22}\end{array}\right)$ is a constant matrix in $(n+m) \times$ $(n+m)$, with $K_{11}$ in $\mathbb{R}^{n \times n}, K_{12}$ in $\mathbb{R}^{n \times m}, K_{21}$ in $\mathbb{R}^{m \times n}$, $K_{22}$ in $\mathbb{R}^{m \times m}$.

Given two functions $y^{0}:[0,1] \rightarrow \mathbb{R}^{n}$ and $z^{0}:[0,1] \rightarrow \mathbb{R}^{m}$, the initial conditions are:

$$
\left(\begin{array}{l}
y(x, 0) \\
z(x, 0)
\end{array}\right)=\left(\begin{array}{l}
y^{0}(x) \\
z^{0}(x)
\end{array}\right), \quad x \in[0,1] .
$$

Remark 1: According to Proposition 2.1 in [14], for all $\left(\begin{array}{l}y^{0} \\ z^{0}\end{array}\right) \in L^{2}(0,1)$, there exists a unique solution $\left(\begin{array}{l}y \\ z\end{array}\right) \in$ $C^{0}\left([0,+\infty), L^{2}(0,1)\right)$ for the Cauchy problem (1)-(3). By Proposition 2.1 in [15], for every $\left(\begin{array}{c}y^{0} \\ z^{0}\end{array}\right) \in H^{2}(0,1)$ satisfying the following compatibility conditions:

$$
\begin{aligned}
\left(\begin{array}{c}
y^{0}(0) \\
z^{0}(0)
\end{array}\right) & =K\left(\begin{array}{c}
y^{0}(1) \\
z^{0}(1)
\end{array}\right), \\
\left(\begin{array}{c}
\Lambda_{1} y_{x}^{0}(0) \\
\varepsilon^{-1} \Lambda_{2} z_{x}^{0}(0)
\end{array}\right) & =K\left(\begin{array}{c}
\Lambda_{1} y_{x}^{0}(1) \\
\varepsilon^{-1} \Lambda_{2} z_{x}^{0}(1)
\end{array}\right),
\end{aligned}
$$

the Cauchy problem (1)-(3) has a unique maximal classical solution $\left(\begin{array}{l}y \\ z\end{array}\right) \in C^{0}\left([0,+\infty), H^{2}(0,1)\right)$.

Let us compute the reduced and boundary-layer subsystems for (1)-(2) adapting the approach of [8] to the infinite dimensional case. By setting $\varepsilon=0$ in system (1), the reduced system is computed from:

$$
\begin{aligned}
y_{t}(x, t)+\Lambda_{1} y_{x}(x, t) & =0, \\
z_{x}(x, t) & =0 .
\end{aligned}
$$

Substituting (6b) into the boundary conditions (2) and assuming $\left(I_{m}-K_{22}\right)$ invertible yields:

$$
\begin{aligned}
y(0, t) & =\left(K_{11}+K_{12}\left(I_{m}-K_{22}\right)^{-1} K_{21}\right) y(1, t), \\
z(., t) & =\left(I_{m}-K_{22}\right)^{-1} K_{21} y(1, t) .
\end{aligned}
$$

The reduced system in $\mathbb{R}^{n}$ is defined as

$$
\bar{y}_{t}(x, t)+\Lambda_{1} \bar{y}_{x}(x, t)=0, \quad x \in[0,1], \quad t \in[0,+\infty),
$$

with the boundary condition

$$
\bar{y}(0, t)=K_{r} \bar{y}(1, t), \quad t \in[0,+\infty),
$$

where $K_{r}=K_{11}+K_{12}\left(I_{m}-K_{22}\right)^{-1} K_{21}$, whereas the initial condition is given as

$$
\bar{y}(x, 0)=y^{0}(x), \quad x \in[0,1] .
$$

To define the boundary-layer system, let us first perform a change of variable

$$
\bar{z}=z-\left(I_{m}-K_{22}\right)^{-1} K_{21} y(1, t),
$$

this shifts the equilibrium of $z$ to the origin.

The boundary-layer system in $\mathbb{R}^{m}$ is defined as

$$
\bar{z}_{\tau}(x, \tau)+\Lambda_{2} \bar{z}_{x}(x, \tau)=0, \quad x \in[0,1], \quad \tau \in[0,+\infty),
$$

with the boundary condition:

$$
\bar{z}(0, \tau)=K_{22} \bar{z}(1, \tau), \quad \tau \in[0,+\infty),
$$

where $\tau=\frac{t}{\varepsilon}$ is a stretching time scale. In $\tau$ time scale, $y(1, t)$ in (10) is handled as a fixed parameter with respect to time.

The initial condition of the boundary-layer system is

$$
\bar{z}(x, 0)=z^{0}(x)-\left(I_{m}-K_{22}\right)^{-1} K_{21} y^{0}(1), \quad x \in[0,1] .
$$

Assuming $\rho_{1}(K)<1$ which implies in particular $I_{m}-$ $K_{22}$ invertible, we next state Tikhonov theorem for linear singularly perturbed system of conservation laws:

Theorem 1: [10] Consider the linear singularly perturbed system of conservation laws (1)-(2). Assume that the boundary conditions matrix $K$ satisfies $\rho_{1}(K)<1$, then, for all initial conditions $y^{0} \in H^{2}(0,1)$ satisfying the compatibility conditions for the reduced system $y^{0}(0)=$ $K_{r} y^{0}(1), \Lambda_{1} y_{x}^{0}(0)=K_{r} \Lambda_{1} y_{x}^{0}(1)$, and $z^{0} \in L^{2}(0,1)$, there exist positive values $\varepsilon^{*}, C, C^{\prime}$ and $\omega$ such that for all $0<\varepsilon<\varepsilon^{*}$ and for all $t \geqslant 0$,

$$
\begin{gathered}
\|y(., t)-\bar{y}(., t)\|_{L^{2}}^{2} \leqslant C \varepsilon e^{-\omega t}, \\
\int_{0}^{\infty}\left\|z(., t)-\left(I_{m}-K_{22}\right)^{-1} K_{21} \bar{y}(1, t)\right\|_{L^{2}}^{2} d t \leqslant C^{\prime} \varepsilon .
\end{gathered}
$$

Proof: The full proof can be seen in [10] and it is based on the analysis of a Lyapunov function for the system which contains the error of slow dynamics between the full system and the reduced system, and the error of the fast dynamics between the full system and its equilibrium point.

\section{BOUNDARY CONTROL SYNTHESIS BASED ON THE SINGULAR PERTURBATION METHOD}

The boundary control synthesis method used in this paper relies on the singular perturbation technique. In the previous section, we have recalled the linear singularly perturbed system (1)-(2). Obviously, the ideal choice of the boundary conditions for the full system is $K=0$. Such boundary conditions make the solutions converge to the equilibrium in finite time. However, in the actual physical problems, the boundary conditions are not always free to be chosen, for instance see the example in Section IV where the structure of boundary conditions matrix is prescribed by physical constraint.

In this section, we consider a singular perturbation approach for the boundary condition synthesis. More precisely, we first choose the boundary conditions matrix $K_{r}$ for the reduced system (7)-(8) as 0 , it makes the slow dynamics converge to the equilibrium in finite time, and the fast dynamics are not modified. For example in Section IV the boundary conditions matrix $K_{r}=0$ for the reduced system is achieved by a suitable choice of the control actions. Then the boundary conditions matrix $K$ for the full system (1)-(2) can be chosen 
based on the boundary conditions for the slow dynamics such that the stability condition $\rho_{1}(K)<1$ for the full system is satisfied.

Before introducing the stability result, let us first give the following definition:

Definition 1: The reduced system (7)-(8) is convergent in finite time if there exists positive value $T$ such that for every initial condition $\bar{y}^{0} \in H^{2}(0,1)$ satisfying the compatibility conditions $y^{0}(0)=K_{r} y^{0}(1), \Lambda_{1} y_{x}^{0}(0)=K_{r} \Lambda_{1} y_{x}^{0}(1)$, the solution to the system (7)-(8) equals zero for all $t \geqslant T$ :

$$
\bar{y}(., t)=0, \quad t \in[T,+\infty) .
$$

Proposition 1: If the boundary conditions matrix $K_{r}=$ 0 , then the reduced system (7)-(8) is convergent in finite time $T$, where $T$ is given by

$$
T=\frac{1}{\lambda_{\min }\left(\Lambda_{1}\right)}
$$

Corollary 1: If the boundary conditions matrix $K_{r}$ for the reduced system (7)-(8) is 0 and if the boundary conditions matrix $K$ for the linear singularly perturbed system of conservation laws (1)-(2) satisfies $\rho_{1}(K)<1$, then, for every initial condition $y^{0} \in H^{2}(0,1)$ satisfying the compatibility conditions $y^{0}(0)=0$ and $y_{x}^{0}(0)=0$, and for all $z^{0} \in$ $L^{2}(0,1)$, there exist positive values $\varepsilon^{*}, C, C^{\prime}, \omega$ and $T=$ $\frac{1}{\lambda_{\min }\left(\Lambda_{1}\right)}$, such that for all $0<\varepsilon<\varepsilon^{*}$ and for all $t \geqslant T$,

$$
\begin{gathered}
\|y(., t)\|_{L^{2}}^{2} \leqslant C \varepsilon e^{-\omega t}, \\
\int_{T}^{\infty}\|z(., t)\|_{L^{2}}^{2} d t \leqslant C^{\prime} \varepsilon .
\end{gathered}
$$

Proof: The proof of this corollary is based on Theorem 1 and Proposition 1. Using Proposition 1 it follows that $\bar{y}(x, t)$ converges to the origin within time $T$, and using (14) in Theorem 1, we get that (17) holds. Moreover it is deduced from (15)

$$
\begin{aligned}
& \int_{0}^{T}\left\|z(., t)-\left(I_{m}-K_{22}\right)^{-1} K_{21} \bar{y}(1, t)\right\|_{L^{2}}^{2} d t \\
+ & \int_{T}^{\infty}\left\|z(., t)-\left(I_{m}-K_{22}\right)^{-1} K_{21} \bar{y}(1, t)\right\|_{L^{2}}^{2} d t \leqslant C^{\prime} \varepsilon,
\end{aligned}
$$

and thus

$$
\int_{T}^{\infty}\left\|z(., t)-\left(I-K_{22}\right)^{-1} K_{21} \bar{y}(1, t)\right\|_{L^{2}}^{2} d t \leqslant C^{\prime} \varepsilon .
$$

Similarly, using Proposition 1 in (19), we get that (18) holds. This concludes the proof of Corollary 1.

This new method for boundary control synthesis is effective. Instead of choosing $K=0$ for the full system, the boundary conditions matrix $K_{r}$ for the reduced system is selected as 0 . The slow dynamics converges to the equilibrium in finite time. The boundary conditions for the full system can be chosen based on that for the reduced system, such that the stability condition $\rho_{1}(K)<1$ is satisfied. Then the full system converges to a small neighborhood of the origin in finite time.

\section{DESIGN OF BOUNDARY CONTROL FOR THE SAINT-VENANT-EXNER MODEL}

In this section, we apply the main result of the previous section to the Saint-Venant-Exner equation which is an example of a singularly perturbed system of conservation laws. We consider a prismatic open channel with a rectangular cross-section and a unit width, where all the friction losses are neglected. The effect of the sediment on the flow is handled in this model. The dynamics of the system are described by the Saint-Venant equation in [16] and Exner equation in [17], [18], [19]:

$$
\begin{aligned}
H_{t}+V H_{x}+H V_{x} & =0 \\
V_{t}+V V_{x}+g H_{x}+g B_{x} & =0, \quad x \in[0,1], \quad t \geqslant 0, \\
B_{t}+a V^{2} V_{x} & =0
\end{aligned}
$$

where the state variables are the water level $H(x, t)$, the water velocity $V(x, t)$, the bathymetry $B(x, t)$ which is the sediment layer above the channel bottom. The gravity constant is $g$ and the constant parameter which represents the porosity and viscosity effects on the sediment dynamics is denoted by $a$. The space variable is $x \in[0,1]$ and the time variable is $t \geqslant 0$.

\section{A. System linearization}

Let us consider a constant in space steady-state $H^{*}, V^{*}$, $B^{*}$. More precisely, (20c) gives $V_{x}^{*}=0$, and we get successively $H_{x}^{*}=0$ and $B_{x}^{*}=0$ from (20a) and (20b). Let us define the deviations of the state $H, V$ and $B$ with respect to the steady-state, for all $x \in[0,1]$ and $t \geqslant 0$,

$$
\begin{aligned}
& h=H-H^{*}, \\
& v=V-V^{*}, \\
& b=B-B^{*} .
\end{aligned}
$$

The linearization of system (20) around the steady-state yields

$$
\begin{aligned}
h_{t}+V^{*} h_{x}+H^{*} v_{x} & =0, \\
v_{t}+g h_{x}+g b_{x}+V^{*} v_{x} & =0, \\
b_{t}+a V^{* 2} v_{x} & =0 .
\end{aligned}
$$

\section{B. Dynamics in Riemann coordinates}

Let us perform a change of variable for the linearized system (21). More precisely, following [18], [19], the characteristic coordinates are defined for each $k=1,2,3$ by

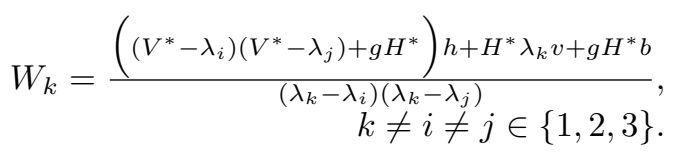

Using the new variables $W_{k}$, system (21) can be rewritten as

$$
W_{t}+\Lambda W_{x}=0
$$

where $W=\left(\begin{array}{lll}W_{1} & W_{2} & W_{3}\end{array}\right)^{T}$ and $\Lambda=$ $\operatorname{diag}\left(\lambda_{1} \quad \lambda_{2} \quad \lambda_{3}\right)$, for all $x \in[0,1], t \in[0,+\infty)$.

According to [18], [19], the three eigenvalues of $\Lambda$ are such that

$$
\lambda_{1}<0<\lambda_{2}<\lambda_{3} .
$$


In [18], [19], $\lambda_{1}$ and $\lambda_{3}$ represent the velocities of the water flow and $\lambda_{2}$ represents the velocity of the sediment motion. The sediment motion is much slower than the water flow, then we get that $\lambda_{2}<<\left|\lambda_{1}\right|$ and $\lambda_{2}<<\lambda_{3}$. In this case by performing the change of spatial variable $W_{1}^{\prime}(1-x, t)=$ $W_{1}(x, t)$, we may assume without loss of generality that $\lambda_{1}>0$, thus $\Lambda$ is diagonal positive.

Let us define a small positive value $\varepsilon=\frac{\lambda_{2}}{\lambda_{3}}$, and a new time scale $\tilde{t}=\lambda_{2} t$, the system (23) is rewritten as the following singularly perturbed system, for all $x \in[0,1]$ and for all $\tilde{t} \geqslant 0$,

$$
\begin{aligned}
\varepsilon W_{1 \tilde{t}}^{\prime}+\frac{\lambda_{1}}{\lambda_{3}} W_{1 x}^{\prime} & =0, \\
W_{2 \tilde{t}}+W_{2 x} & =0, \\
\varepsilon W_{3 \tilde{t}}+W_{3 x} & =0 .
\end{aligned}
$$

\section{Boundary conditions}

We assume that the channel is equipped with hydraulic control devices such as pumps, valves, spillways, gates, etc. The water levels at upstream and downstream of the channel are assumed to be measured. The control action is provided by the control devices. In the present paper, we introduce the following three boundary conditions (these are the same boundary conditions as in [20]):

1) The first boundary condition describes the value of the channel inflow rate which is denoted by $c_{0}(\tilde{t})$. Here we consider $c_{0}(\tilde{t})$ as a control input (see [21]):

$$
H(0, \tilde{t}) V(0, \tilde{t})=c_{0}(\tilde{t}) .
$$

2) The second boundary condition is given by gate operation at outflow of the reach. A gate model can be expressed as follows (see [21]):

$$
H(1, \tilde{t}) V(1, \tilde{t})=\alpha \sqrt{\left[H(1, \tilde{t})-c_{1}(\tilde{t})\right]^{3}},
$$

where $\alpha$ is a positive constant coefficient. The control input is denoted by $c_{1}(\tilde{t})$.

3) The third boundary condition is a physical constraint on the bathymetry (see [19]):

$$
B(0, \tilde{t})=\mathcal{B}
$$

where $\mathcal{B}$ is a constant value.

After the linearization of these boundary conditions, we derive the following boundary conditions for system (21):

$$
\begin{aligned}
H^{*} v(0, \tilde{t})+V^{*} h(0, \tilde{t}) & =c_{0}(\tilde{t})-c_{0}^{*}, \\
H^{*} v(1, \tilde{t})+V^{*} h(1, \tilde{t}) & =\frac{3 \alpha\left(h(1, \tilde{t})+c_{1}^{*}-c_{1}(\tilde{t})\right) \sqrt{H^{*}-c_{1}^{*}}}{2} \\
b(0, \tilde{t}) & =0
\end{aligned}
$$

where $c_{0}^{*}$ and $c_{1}^{*}$ are constant control actions at the steadystate $\left(\begin{array}{lll}H^{*} & V^{*} & B^{*}\end{array}\right)^{T}$.

The boundary conditions for system (25) are given as follows:

$$
\begin{aligned}
& W_{1}^{\prime}(0, \tilde{t})=k_{12} W_{2}(1, \tilde{t})+k_{13} W_{3}(1, \tilde{t}), \\
& W_{2}(0, \tilde{t})=k_{21} W_{1}^{\prime}(1, \tilde{t}), \\
& W_{3}(0, \tilde{t})=\xi\left(k_{21}\right) W_{1}^{\prime}(1, \tilde{t}),
\end{aligned}
$$

with

$$
\xi\left(k_{21}\right)=-\frac{\left[\left(\lambda_{1}-V^{*}\right)^{2}-g H^{*}\right]+k_{21}\left[\left(\lambda_{2}-V^{*}\right)^{2}-g H^{*}\right]}{\left(\lambda_{3}-V^{*}\right)^{2}-g H^{*}} .
$$

Proposition 2: The boundary conditions (26)-(28) for the system (21) are equivalent to the boundary conditions (29)(31) for the system (25) with the following boundary control inputs, for all $\tilde{t} \geqslant 0$,

$$
\begin{aligned}
& c_{0}(\tilde{t})=c_{0}^{*}+h(0, \tilde{t})\left[V^{*}+\frac{g H^{*}\left(k_{21} \phi_{1}-\phi_{2}\right)}{\phi_{2} \lambda_{2}-k_{21} \phi_{1} \lambda_{1}}\right. \\
& \left.+\frac{k_{21} \phi_{1}\left(V^{*}-\lambda_{2}\right)\left(V^{*}-\lambda_{3}\right)-\phi_{2}\left(V^{*}-\lambda_{1}\right)\left(V^{*}-\lambda_{3}\right)}{\phi_{2} \lambda_{2}-k_{21} \phi_{1} \lambda_{1}}\right],
\end{aligned}
$$

$$
\begin{aligned}
& c_{1}(\tilde{t})=c_{1}^{*}+h(1, \tilde{t})\left[1-\frac{2 V^{*}}{3 \alpha \sqrt{H^{*}-c_{1}^{*}}}\right. \\
& -\frac{2 H^{*}}{3 \alpha \sqrt{H^{*}-c_{1}^{*}}} \times\left(\frac{g H^{*}\left(-\phi_{1}+k_{12} \phi_{2}+k_{13} \phi_{3}\right)}{H^{*}\left(\lambda_{1} \phi_{1}-k_{12} \lambda_{2} \phi_{2}-k_{13} \lambda_{3} \phi_{3}\right)}\right. \\
& -\frac{\phi_{1}\left(V^{*}-\lambda_{2}\right)\left(V^{*}-\lambda_{3}\right)-k_{12} \phi_{2}\left(V^{*}-\lambda_{1}\right)\left(V^{*}-\lambda_{3}\right)}{H^{*}\left(\lambda_{1} \phi_{1}-k_{12} \lambda_{2} \phi_{2}-k_{13} \lambda_{3} \phi_{3}\right)} \\
& \left.\left.+\frac{k_{13} \phi_{3}\left(V^{*}-\lambda_{1}\right)\left(V^{*}-\lambda_{2}\right)}{H^{*}\left(\lambda_{1} \phi_{1}-k_{12} \lambda_{2} \phi_{2}-k_{13} \lambda_{3} \phi_{3}\right)}\right)\right] \\
& -b(1, \tilde{t})\left[\frac{2 H^{*}}{3 \alpha \sqrt{H^{*}-c_{1}^{*}}} \frac{g\left(-\phi_{1}+k_{12} \phi_{2}+k_{13} \phi_{3}\right)}{\lambda_{1} \phi_{1}-k_{12} \lambda_{2} \phi_{2}-k_{13} \lambda_{3} \phi_{3}}\right],
\end{aligned}
$$

where $H^{*}-c_{1}^{*} \neq 0$ and $\phi_{k}=\frac{1}{\left(\lambda_{k}-\lambda_{i}\right)\left(\lambda_{k}-\lambda_{j}\right)}$ for $(i, j, k)$ in $\{1,2,3\}^{3}$.

Adopting the definitions of the reduced system and the boundary-layer system in Section II, the two subsystems are computed as follows. The reduced system is

$$
\bar{W}_{2 \tilde{t}}+\bar{W}_{2 x}=0
$$

with the boundary condition

$$
\bar{W}_{2}(0, \tilde{t})=K_{r} \bar{W}_{2}(1, \tilde{t})
$$

where $K_{r}=\frac{k_{12} k_{21}}{1-k_{13} \xi\left(k_{21}\right)}$.

Let us perform the following change of variables:

$$
\begin{aligned}
& \bar{W}_{1}^{\prime}=W_{1}^{\prime}-\frac{k_{12}}{1-k_{13} \xi\left(k_{21}\right)} W_{2}(1, \tilde{t}), \\
& \bar{W}_{3}=W_{3}-\frac{k_{12} \xi\left(k_{21}\right)}{1-k_{13} \xi\left(k_{21}\right)} W_{2}(1, \tilde{t}) .
\end{aligned}
$$

The boundary-layer system is

$$
\left(\begin{array}{l}
\bar{W}_{1}^{\prime} \\
\bar{W}_{3}
\end{array}\right)_{\tilde{\tau}}+\left(\begin{array}{cc}
\frac{\lambda_{1}}{\lambda_{3}} & 0 \\
0 & 1
\end{array}\right)\left(\begin{array}{l}
\bar{W}_{1}^{\prime} \\
\bar{W}_{3}
\end{array}\right)_{x}=0
$$

with the boundary conditions

$$
\left(\begin{array}{l}
\bar{W}_{1}^{\prime}(0, \tilde{\tau}) \\
\bar{W}_{3}(0, \tilde{\tau})
\end{array}\right)=K_{22}\left(\begin{array}{l}
\bar{W}_{1}^{\prime}(1, \tilde{\tau}) \\
\bar{W}_{3}(1, \tilde{\tau})
\end{array}\right)
$$

where $K_{22}=\left(\begin{array}{cc}0 & k_{13} \\ \xi\left(k_{21}\right) & 0\end{array}\right), \tilde{\tau}=\frac{\tilde{t}}{\varepsilon}$. 


\section{Boundary control synthesis}

The boundary conditions matrix $K_{r}$ for the reduced system (35)-(36) need to be chosen as 0 . Assuming that $1-k_{13} \xi\left(k_{21}\right) \neq 0, K_{r}=0$ holds as soon as $k_{12}=0$ or $k_{21}=0$.

Proposition 3: Consider the boundary conditions matrix

$$
K_{1}=\left(\begin{array}{ccc}
0 & k_{12} & k_{13} \\
0 & 0 & 0 \\
\xi(0) & 0 & 0
\end{array}\right)
$$

with $k_{12}, k_{13}$ in $\mathbb{R}$, assume that $k_{13} \xi(0) \neq 1$ and that there exist positive values $d_{2}, d_{3}$ such that

$$
\left(\begin{array}{cccccc}
1 & 0 & 0 & 0 & 0 & d_{3} \xi(0) \\
0 & d_{2} & 0 & k_{12} & 0 & 0 \\
0 & 0 & d_{3} & k_{13} & 0 & 0 \\
0 & k_{12} & k_{13} & 1 & 0 & 0 \\
0 & 0 & 0 & 0 & d_{2} & 0 \\
\xi(0) & 0 & 0 & 0 & 0 & d_{3}
\end{array}\right)>0
$$

is satisfied. Consider the boundary conditions matrix

$$
K_{2}=\left(\begin{array}{ccc}
0 & 0 & k_{13} \\
k_{21} & 0 & 0 \\
\xi\left(k_{21}\right) & 0 & 0
\end{array}\right),
$$

with $k_{21}, k_{13}$ in $\mathbb{R}$, assume that $k_{13} \xi\left(k_{21}\right) \neq 1$ and that there exist positive values $d_{2}, d_{3}$ such that

$$
\left(\begin{array}{cccccc}
1 & 0 & 0 & 0 & k_{21} d_{2} & \xi\left(k_{21}\right) d_{3} \\
0 & d_{2} & 0 & 0 & 0 & 0 \\
0 & 0 & d_{3} & k_{13} & 0 & 0 \\
0 & 0 & k_{13} & 1 & 0 & 0 \\
k_{21} d_{2} & 0 & 0 & 0 & d_{2} & 0 \\
\xi\left(k_{21}\right) d_{3} & 0 & 0 & 0 & 0 & d_{3}
\end{array}\right)>0
$$

is satisfied. Then Corollary 1 can be applied to system (25) with either boundary conditions matrix $K_{1}$ or $K_{2}$ defining the boundary conditions (29)-(31).

To solve (41), we can compute $\xi(0)$ from (32), then (41) is a linear matrix inequality (LMI) which can be solved. Similarly, by choosing $\xi\left(k_{21}\right)=0$ in (43), $k_{21}$ is computed from (32), then LMI (43) can be solved.

\section{E. Numerical simulation}

Using the numerical values in [22], the equilibrium is chosen as $H^{*}=0.1365, V^{*}=14.65, B^{*}=0$. We take the gravity constant $g=9.81$. The eigenvalues of matrix $\Lambda$ are also given in [22] as $\lambda_{1}=-10, \lambda_{2}=7.72 \times 10^{-4}$, $\lambda_{3}=13$. Using Yalmip toolbox [23] on Matlab to solve LMI (41) and (43). The obtained boundary conditions matrix $K_{1}$ is

$$
K_{1}=\left(\begin{array}{ccc}
0 & 1 & 0 \\
0 & 0 & 0 \\
-14 & 0 & 0
\end{array}\right)
$$

and $K_{2}$ is

$$
K_{2}=\left(\begin{array}{ccc}
0 & 0 & 0 \\
0.095 & 0 & 0 \\
0 & 0 & 0
\end{array}\right)
$$

To numerically compute the solutions of system (25) with the boundary conditions matrix (44) or (45), we discretize them by using a two-step variant of the Lax-Wendroff method (see [24] and [25]). Precisely, the space domain [0,1] is divided into 100 intervals of identical length, the final time is chosen as 2000. We take a time-step that satisfies the CFL condition and select the initial conditions as follows, for all $x \in[0,1]$,

$$
\begin{aligned}
& W_{1}^{\prime 0}(x)=-1+\cos (4 \pi x), \\
& W_{2}^{0}(x)=-1+\cos (2 \pi x), \\
& W_{3}^{0}(x)=1-\cos (4 \pi x) .
\end{aligned}
$$

Fig. 1 shows the dynamics $\bar{W}_{2}$ for the reduced system (35). It converges to the origin within time $T \simeq 1300 \mathrm{~s}$ for the boundary condition matrix $K_{r}=0$. The finite time of convergence obtained in Proposition 1 is $T=\frac{1}{\lambda_{2}}$ which is close to the numerically computed finite time $T \simeq 1300 \mathrm{~s}$. The slow dynamics $W_{2}$ for system (25) with the boundary conditions matrix $K_{1}$ given by (44) in Fig. 2 is roughly the same graph as $\bar{W}_{2}$ in Fig. 1. Fig. 3-4 show the time evolutions of the fast dynamics for system (25) with the boundary conditions matrix $K_{1}$. It is observed that the solutions converge to 0 as time increases and that they depend on the evolution of the slow dynamics.

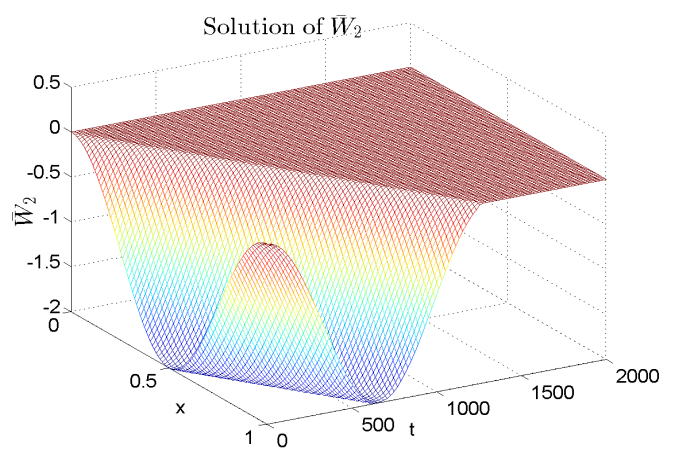

Fig. 1: Time evolution of $\bar{W}_{2}$ for system (35) with $K_{r}=0$

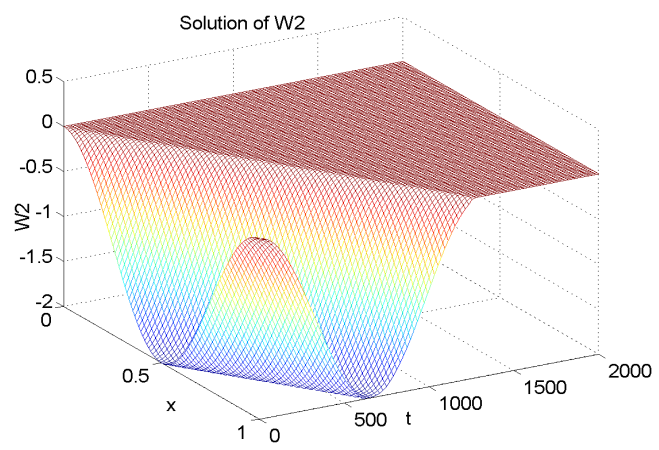

Fig. 2: Time evolution of the slow dynamics $W_{2}$ for system (25) with $K_{1}$ 


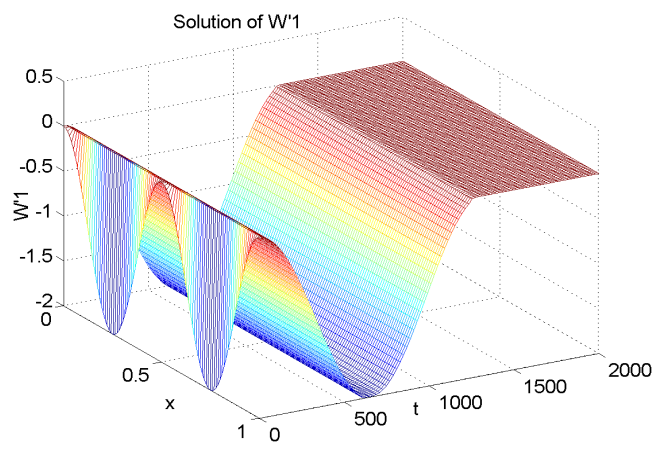

Fig. 3: Time evolution of the fast dynamics $W_{1}^{\prime}$ for system (25) with $K_{1}$

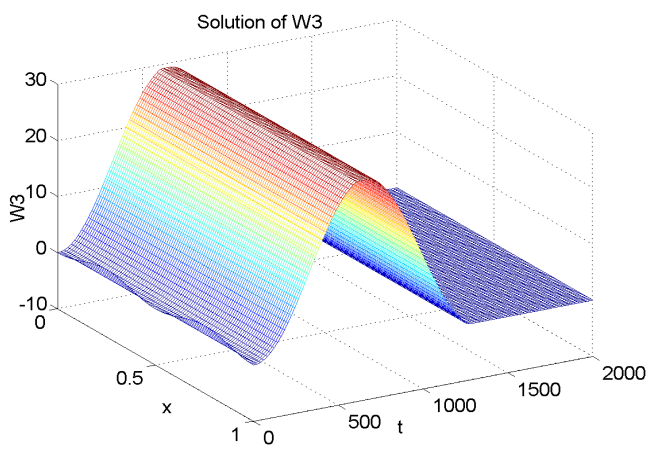

Fig. 4: Time evolution of the fast dynamics $W_{3}$ for system (25) with $K_{1}$

Similar results are obtained for system (25) with the boundary conditions matrix $K_{2}$ by numerical simulations.

\section{CONCLUSIONS}

In this paper, the boundary control synthesis of a class of linear hyperbolic systems has been studied based on the singular perturbation method. The slow dynamics is stabilized within time $T$ by choosing the boundary conditions for the reduced system as zero. The main result is applied to a boundary control design for a hyperbolic system represented by the Saint-Venant-Exner equation. The simulation example shows the effectiveness of the contribution of this work.

This work could be applied to different kinds of physical systems governed by singularly perturbed systems of conservation laws, such as the flow control in [26]. These applications will be considered in the future works.

\section{REFERENCES}

[1] G. Bastin, J.-M. Coron, and B. d'Andréa Novel. Using hyperbolic systems of balance laws for modeling, control and stability analysis of physical networks. In Lecture notes for the Pre-Congress Workshop on Complex Embedded and Networked Control Systems, Seoul, Korea, 2008. 17th IFAC Word Congress.
[2] M. Dick, M. Gugat, and G. Leugering. Classical solutions and feedback stabilization for the gas flow in a sequence of pipes. Networks and Heterogeneous Media, 5(4):691-709, 2010.

[3] M. Gugat. Boundary feedback stabilization of the telegraph equation: Decay rates for vanishing damping term. Systems and Control Letters, 66:72-84, 2014.

[4] B. Haut and G. Bastin. A second order model of road junctions in fluid models of traffic networks. Networks and Heterogeneous Media, 2(2):227-253, 2007.

[5] M. Gugat and G. Leugering. Global boundary controllability of the Saint-Venant system for sloped canals with friction. Annales de l'Institut Henri Poincare (C) Non Linear Analysis, 26(1):257-270, 2009.

[6] M. Gugat, M. Herty, A. Klar, and G. Leugering. Optimal control for traffic flow networks. Journal of Optimization Theory and Applications, 126(3):589-616, 2005.

[7] R.M. Colombo, G. Guerra, M. Herty, and V. Schleper. Optimal control in networks of pipes and canals. SIAM Journal on Control and Optimization, 48:2032-2050, 2009.

[8] P. Kokotović, H.K. Khalil, and J. O'Reilly. Singular pertrubation methods in control: analysis and design. Academic Press, 1986.

[9] M.K. Kadalbajoo and K.C. Patidar. Singularly perturbed problems in partial differential equations: a survey. Applied Mathematics and Computation, 134:371-429, 2003.

[10] Y. Tang, C. Prieur, and A. Girard. Tikhonov theorem for linear hyperbolic systems. Submitted for publication, 2013. http:// hal. archives-ouvertes.fr/hal-01064805.

[11] M. Krstic and A. Smyshlyaev. Backstepping boundary control for first-order hyperbolic PDEs and application to systems with actuator and sensor delays. Systems and Control Letters, 57(750-758), 2008.

[12] C. Prieur and J. de Halleux. Stabilization of a 1-D tank containing a fluid modeled by the shallow water equations. Systems and Control Letters, 52(3-4):167-178, 2004.

[13] C. Prieur, J. Winkin, and G. Bastin. Robust boundary control of systems of conservation laws. Math. Control Signals System, 20(2):173-197, 2008.

[14] J-M. Coron, G. Bastin, and B. d'Andréa-Novel. Dissipative boundary conditions for one-dimensional nonlinear hyperbolic systems. SIAM Journal on Control and Optimization, 47(3):1460-1498, 2008.

[15] J-M. Coron. Control and nonlinearity, volume 136 of Mathematical Surveys and Monographs. Americam Mathematical Society, 2007.

[16] W.H. Graf. Fluvial hydraulics. Wiley, 1998.

[17] W.H. Graf. Hydraulics of sediment transport. Water Resources Publications, Highlands Ranch, Colorado, USA, 1984.

[18] J. Hudson and P.K. Sweby. Formulations for numerically approximating hyperbolic systems governing sediment transport. Journal of Scientific Computing, 19:225-252, 2003.

[19] A. Diagne, G. Bastin, and J-M. Coron. Lyapunov exponential stability of 1-D liear hyperbolic systems of balance laws. Automatica, 48:109114, 2012.

[20] C. Prieur and F. Mazenc. ISS-Lyapunov functions for time-varying hyperbolic systems of balance laws. Math. Control Signals System, 24(1):111-134, 2012.

[21] G. Bastin, J.-M. Coron, and B. d'Andréa Novel. On Lyapunov stability of linearised Saint-Venant equations for a sloping channel. Networks and Heterogeneous Media, 4(2):177-187, 2009.

[22] V. Dos Santos and C. Prieur. Boundary control of open channels with numerical and experimental validations. IEEE Trans. Control Syst. Tech., 16(6):1252-1264, 2008.

[23] J. Löfberg. Yalmip: A toolbox for modeling and optimization in MATLAB. In In Proc. of the CACSD Conference, Taipei, Taiwan, 2004

[24] L.F. Shampine. Solving hyperbolic PDEs in Matlab. Appl. Numer. Anal. Comput. Math., 2:346-358, 2005.

[25] L.F. Shampine. Two-step Lax-Friedrichs method. Applied Mathematics Letters, 18:1134-1136, 2005.

[26] F. Castillo, E. Witrant, and L. Dugard. Contrôle de Température dans un Flux de Poiseuille. IEEE Conférence Internationale Francophone d'Automatique, Grenoble, France, 2012. 